\title{
Development of a Two-Channel Simultaneous Photoplethysmography Recording System
}

\author{
Kim Soon Chong ${ }^{1}$, Kok Beng $\operatorname{Gan}^{2} \&$ Mohd. Ali Mohd. Alauddin ${ }^{1,2}$ \\ ${ }^{1}$ Department of Electrical, Electronic \& Systems Engineering, Faculty of Engineering \& \\ Built Environment, University Kebangsaan Malaysia, 43600 UKM Bangi, Malaysia. \\ ${ }^{2}$ Institute of Space Science, Universiti Kebangsaan Malaysia, \\ 43600 UKM Bangi, Malaysia \\ Email: gankokbeng@ukm.my
}

\begin{abstract}
Multi-site photoplethysmograph (PPG) recording enables researchers to study the vascular and hemodynamic properties of human subjects. Currently, there is no commercial system available in the market to perform either onechannel or multi-channel PPG recording. PPG is an optoelectronic technique that measures changes in blood volume associated with cardiac contraction. The measurements can be obtained from fingertips, ear lobes and toes due to their low absorption and high degree of vasculature. The main objective of this project is to develop a two-channel simultaneous PPG recording system to acquire PPG signals from two different physiological sites using a Nellcor equivalent PPG probe. MATLAB software was used during the development phase to ensure rapid prototyping. The experiment results show that there was no inter-channel delay in the developed two-channel PPG system. Our preliminary results show that the delays between the left and the right arm were from 4 to $12 \mathrm{~ms}$ in three healthy random subjects. The system is portable, powered by universal serial bus (USB) and allows the user to do the PPG data acquisition in a clinical setting.
\end{abstract}

Keywords: cardiovascular risk; multi-site; non-invasive; photoplethysmograph; pulse wave transit time; simultaneous acquisition.

\section{$1 \quad$ Introduction}

In recent years, the study of the cardiovascular system [1-4] of individuals from an early age has been given an increasing social importance. It has been demonstrated that most heart and vessel diseases are initiated either genetically or induced by lifestyle. Furthermore, ageing is a natural process in humans, which currently is heavily modified by environmental influences [1]. Therefore, the vascular age of an individual may differ from his or her chronological age. Ageing has been defined as a gradual loss of organ functions, especially in the cardiovascular system, where the major effect of ageing is a decrease of vessel compliance in the arterial system. According to the World Health Organization (WHO), cardiovascular diseases (CVD) were one of the top killer diseases in Malaysia during the year 2009 [5]. The main cause of CVD is atherosclerosis

Received June $23^{\text {rd }}, 2012$, Revised September $26^{\text {th }}, 2012$, Accepted for publication September $27^{\text {th }}, 2012$. Copyright (C) 2012 Published by LPPM ITB, ISSN: 1978-3086, DOI: 10.5614/itbj.ict.2012.6.2.5 
where fat or plaque is accumulating on the artery walls of blood vessels [6]. The accumulation of fat or plaque will cause the formation of lesions that eventually grow and thicken to narrow the arteries, which slows blood flow [7], and finally the blood vessels will lose their rigidity and elasticity.

PPG is an optoelectronic method for measuring and recording changes in the volume of body parts such as fingers and ear lobes caused by changes in the volume of the arterial oxygenated blood associated with cardiac contraction [8]. When light travels through biological tissue (earlobe, toe or finger), it is absorbed by different substances [9]. Primary absorbers are skin pigmentation, bones and arterial and venous blood. Arterial ageing and arterial disease influence the characteristics of the PPG pulse [10]. The simplest PPG probe consists of a light emitting diode (LED) and a photo-detector. The LED transmits light with an intensity that is proportional to the LED drive current. The photo-detector is usually a silicon photodiode that produces a current proportional to the incident light on it. Improved technology in photodiodes and LEDs allow the LED and photodiode to be small enough to fit in small fingertip probes. The probes can be of the transmission or the reflection type.

The PPG signal can be affected by various physiological factors, so analysis of the PPG signal can provide sufficient information about the individual's health condition and more specifically about cardiovascular performance [11]. The higher-frequency components $(0.5 \mathrm{~Hz}-30 \mathrm{~Hz})$ in PPG pulses from the left and the right side of normal subjects are highly correlated [12]. The results of this research show that pulse timing, amplitude and shape of the higher-frequency components of the PPG waveforms are similar at the right and the left side of the body in three different sites (fingers, toes and earlobes) since their anatomical structure is similar. Therefore, any difference in vessel properties can affect the time and shape of the rising edge (anacrotic phase) and falling edge (catacrotic phase) of the PPG signal, which can indicate pathological changes $[12,13]$. A time delay between PPG signals from the left and the right arm is evidence of an increase in vascular resistance. A delay between pulses from right and left toes in healthy individuals and patients with some occlusion in the leg has also been reported $[3,13,14]$. This delay was found to be 20 to 80 ms for patients with arterial stenosis in one leg [14]. This group also had an increased pulse wave transit time (PWTT) due to higher vascular resistance. The PWTT is the time for a pulse to travel between two artery sites. The speed at which the arterial pressure wave travels is directly proportional to the blood pressure. An acute rise in blood pressure causes the vascular tone to increase and the arterial wall to become stiffer, and shortens the PWTT.

The main problem with multi-site PPG is the data acquisition process. This instrument has always been custom-made, application specific and developed 
by the researchers $[3,12,15,16]$. Normally, rapid prototyping tools and a data acquisition system such as Virtual Instrumentation (National Instruments Corp.) are used to fabricate a multichannel PPG system [12,16]. This laboratory prototype is suitable for research purposes but not for commercialization or clinical trials. Furthermore, no such commercial devices are available in the market and if they exist it is only as an option for the device. Until now, the acquisition of two-channel PPG signals has been done using two independent PPG systems, a synchronization system and a personal computer [15]. This introduces an uncontrollable artificial delay because the data acquisition is started manually. A possible solution is to use a Barker sequence code to synchronize the recorded PPG signals using offline signal processing [15]. The synchronization of multi-channel PPG signals (Figure 1) may eliminate the unwanted delay in the PWTT but the apparatus of the recording signal is still complex and its mobility is limited [15].

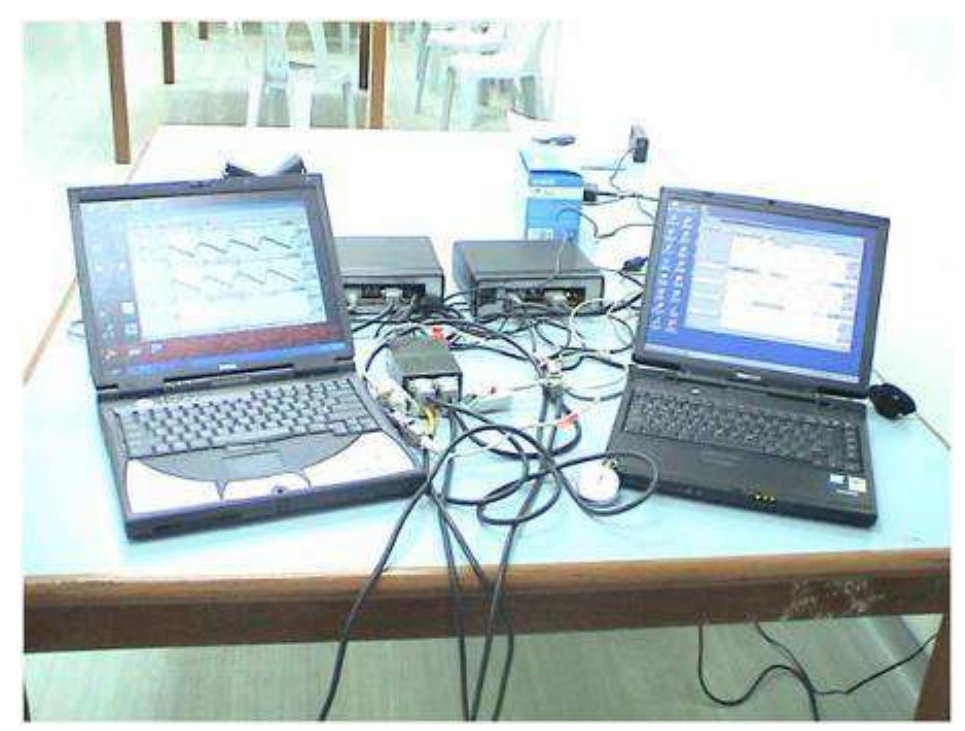

Figure 1 Apparatus for two-channel PPG recording using two independent systems.

In this research, a two-channel simultaneous PPG system was designed and developed to acquire PPG signals simultaneously from two physiological sites. The system simplifies the PPG data acquisition process by using a 16-bit twochannel analog-to-digital converter (ADC). It digitizes and samples the PPG signals simultaneously from two different physiological sites, namely, the index finger of the left and of the right hand. Both signals will then be transferred to a personal computer via USB for further analysis using MATLAB (The MathWorks, Inc.). 


\section{$2 \quad$ Material and Methods}

\subsection{Analog Design}

A basic block diagram of the two-channel PPG system is shown in Figure 2. It consists of an analog and a digital design. In the analog design, the PPG signals are obtained by using two PPG probes (DS-100A, Covidien). The infrared LED $(910 \mathrm{~nm})$ is driven by a LED driver (LT1932, Linear Technology Inc.) at 100 $\mathrm{mA}$. The main function of the LED driver is to maintain the intensity of the infrared LED in the probes so that the intensity is not affected by the power supply. The light is transmitted through the finger and detected by the photodiode located at the opposite side of the LED. A trans-impedance amplifier (OP474) is used to convert the detected current into voltage.

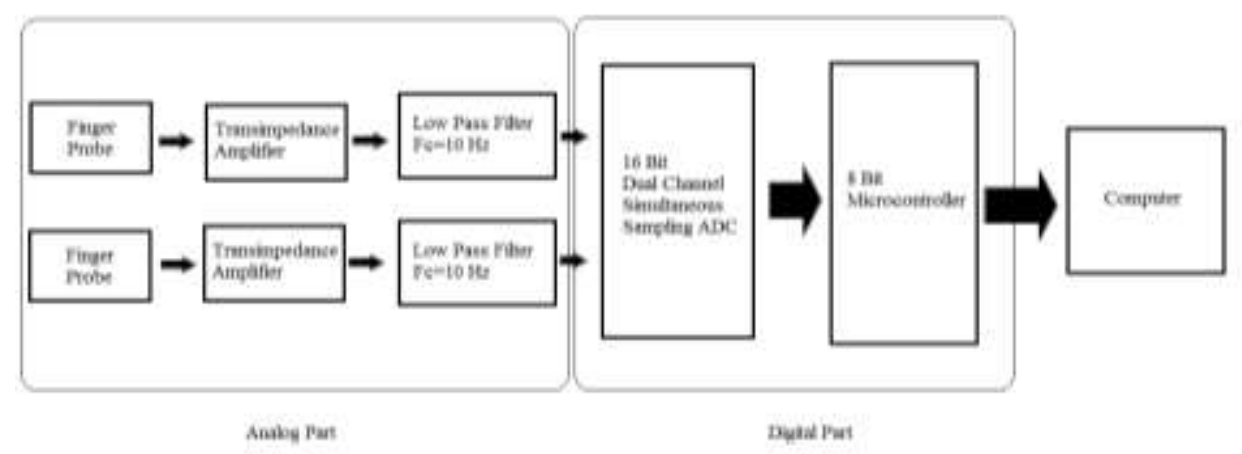

Figure 2 Basic block diagram of the two-channel PPG system.

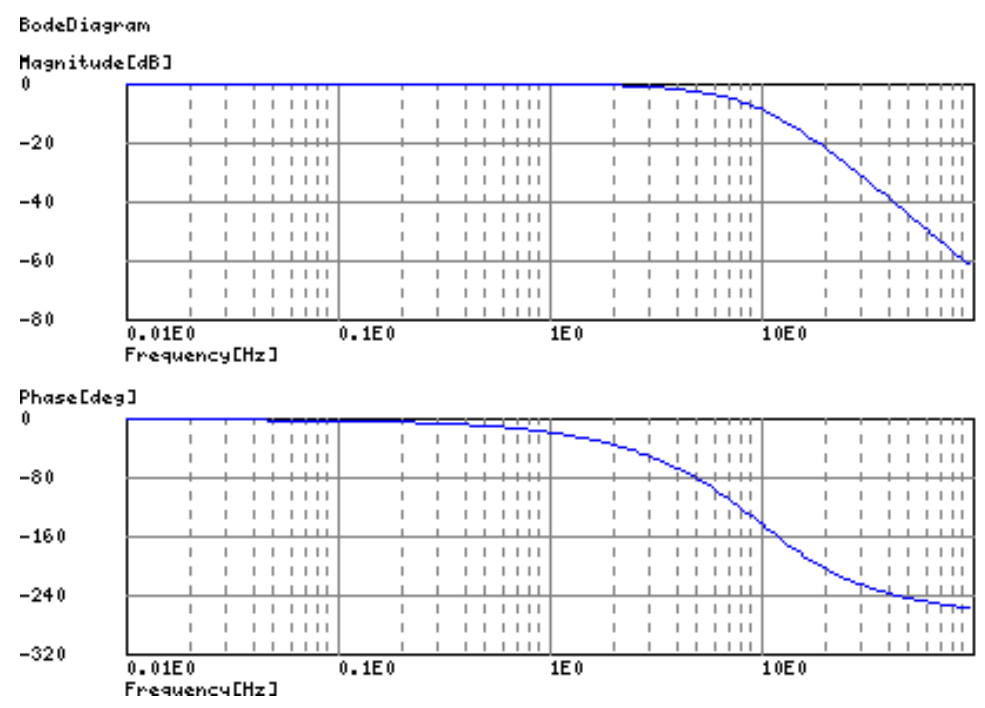

Figure 3 Bode diagram of the second-order Sallen Key Filter. 
The unwanted high frequency noise in the PPG signal is eliminated using a second-order Sallen Key lowpass filter with a cutoff frequency of $10 \mathrm{~Hz}$ at -3 $\mathrm{dB}$. The cutoff frequency of $10 \mathrm{~Hz}$ was chosen considering the frequency content of the PPG signal $(0.6-30 \mathrm{~Hz})$ and because it is far removed from the 50 $\mathrm{Hz}$ power line noise, even at the transition band (Figure 3). The presence of the $50 \mathrm{~Hz}$ power line noise does affect data visualization during data acquisition.

\subsection{Digital Design}

In the digital design, the analog PPG signal is converted into digital format using a 16-bits dual channel ADS8361 ADC. One of the applications of this instrument is to detect changes in vascular stiffness. The physiological delay between two physiological sites in healthy subjects is normally below $20 \mathrm{~ms}$ [11]. The PPG signals from the left and the right index finger are sampled at 2 $\mathrm{kHz}$ so that the physiological delay can be observed. The sampling process is performed using simultaneous ADC sampling to avoid any artificial delay due to the sampling process.

The digitized PPG signal is sent to a 8-bits microcontroller (PIC18F2550) and communicates with a computer via USB. The role of the microcontroller is to control the ADC sampling process and get the data from the ADC. Subsequently, the digitized data are processed and sent to a computer using USB communication. The firmware was developed using $\mathrm{C}$ language and compiled using PICC (CCS-C, Inc). The USB driver was installed in the operating system to establish the communication between the microcontroller and the computer.

\subsection{Data Acquisition Software}

The graphical user interface (GUI) and signal processing of the system was developed using MATLAB R2012b (The MathWorks, Inc.). This GUI was used to display the acquired PPG signals and save them in a text file for preprocessing at a later stage. The PPG signals were plotted in real-time for viewing and signal quality control.

A digital signal processing algorithm was developed to pre-process the PPG signals (Figure 3). This algorithm consists of channel separation, detrending, band-pass filter, scale-to-one and finally determination of the physiological delay using the cross-correlation method.

The serial data transfer protocol was used to transfer the digital data from the ADC to the microcontroller. Therefore, a channel separation algorithm is needed to separate the acquired signals into two-channel PPG signals. 
Subsequently, the linear trend of the PPG signals is removed by detrending the input signal, i.e. the mean value is subtracted from the data.

The signal is further processed to remove the effect of the respiratory rhythm and higher frequency disturbance by applying a band-pass filter at a frequency range of $0.6-15 \mathrm{~Hz}$. This is an effective spectrum for PPG signals used for physiological signal modeling. The band-pass filter is a custom-designed digital filter using the Fast Fourier Transform method. The unwanted frequency band is masked with zeroes before performing the Inverse Fast Fourier Transform to reconstruct the time-domain PPG signals. The pre-processed signal then undergoes a scale-to-one process where the signal is normalized to unity (range 0 to 1). The cross-correlation function of MATLAB is used to determine the time delay between the PPG signals acquired from the left and the right arm in 5 -second blocks. The estimated delay is given by the negative of the lag for which the normalized cross-correlation has the largest absolute value.

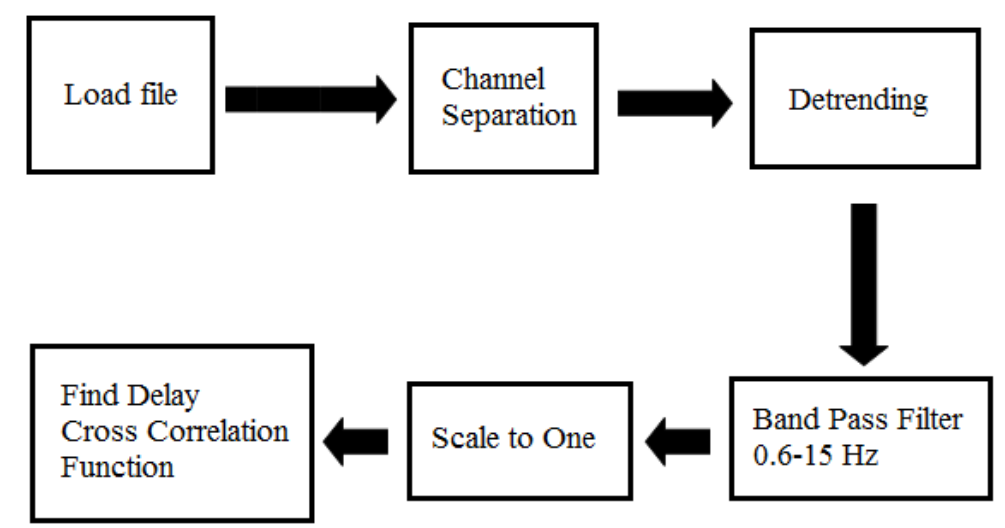

Figure 4 Pre-processing block diagram to find the delay between two-channel PPG signals.

\section{$3 \quad$ Results and Discussions}

\subsection{Two-Channel PPG Recording System}

The schematic of the two-channel PPG recording systems is shown in Figure 4. This was turned into a double-layer printed circuit board (PCB). In this system, the PCB board was partitioned into an analog and a digital section to avoid interference of analog and digital signals. The digital logic ground currents remain in the digital section and will not interfere with the analog currents. Besides that, the location of the passive components could also affect the noise level. For example, the decoupling capacitor should be placed as near as 
possible to the integrated circuit (IC) to effectively reduce the noise in the circuit. Figure 5 shows the final prototype of the two-channel PPG system. This system comprises of two probes and a data acquisition module. Its power source is the USB port and it communicates with the computer using high speed (12 Mbps) USB communication.

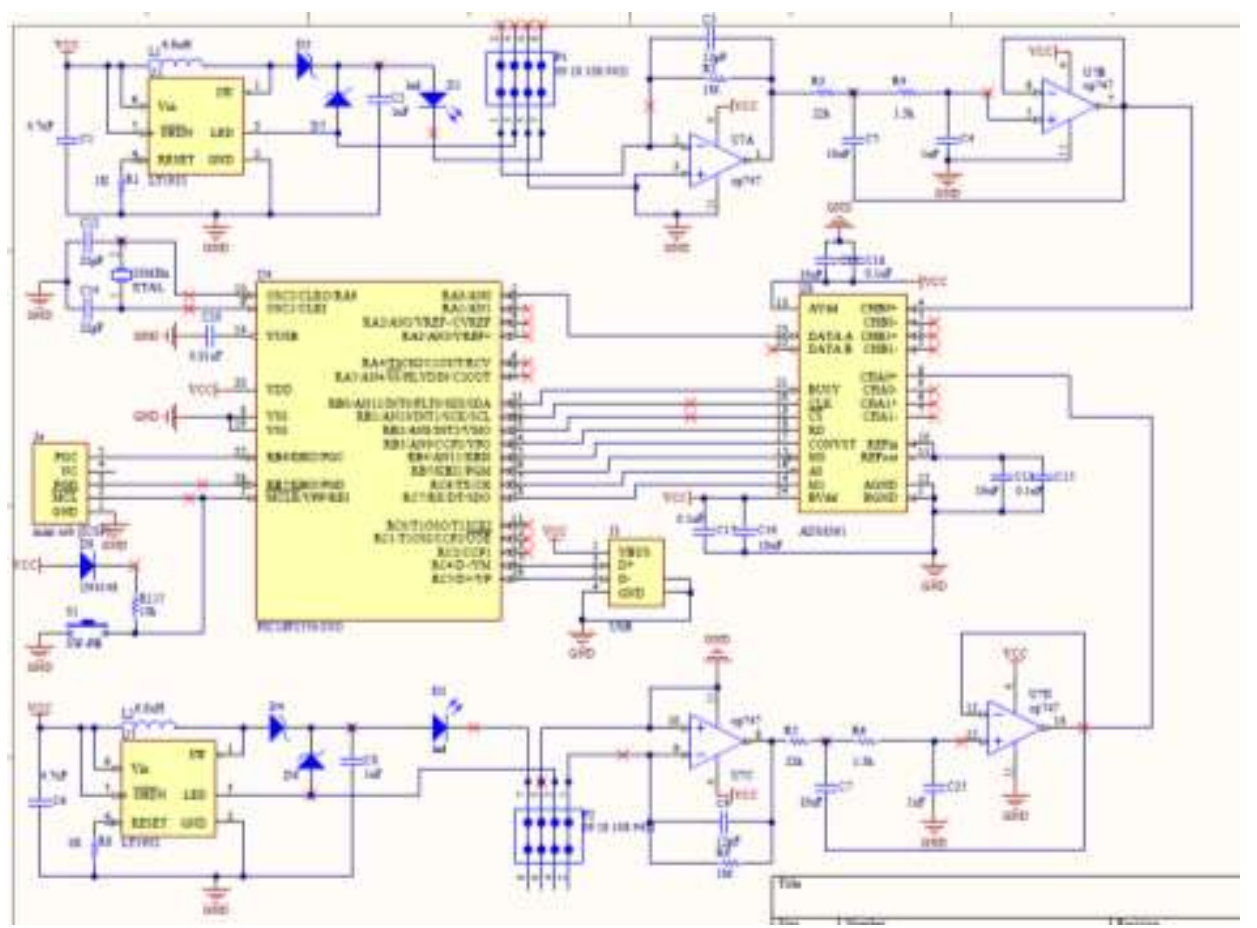

Figure 5 The schematic of the two-channel PPG.

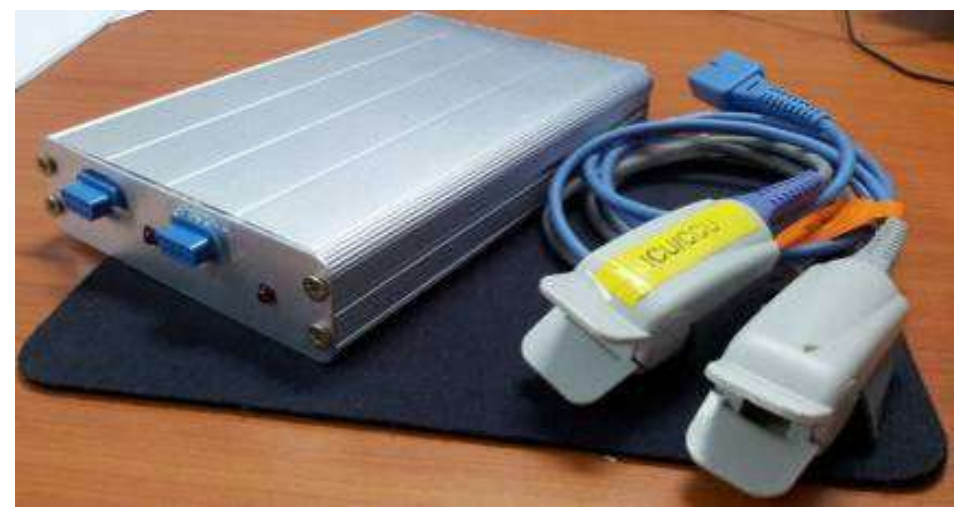

Figure 6 Two-channel PPG system. 
The frequency response of the second-order low-pass filter is shown in Figure 6. Note that the response in the passband was flat with no ripple. The power roll-off approaches $36 \mathrm{~dB}$ per decade in the limit of high frequency. This filter design effectively attenuated the power line noise at $50 \mathrm{~Hz}$.

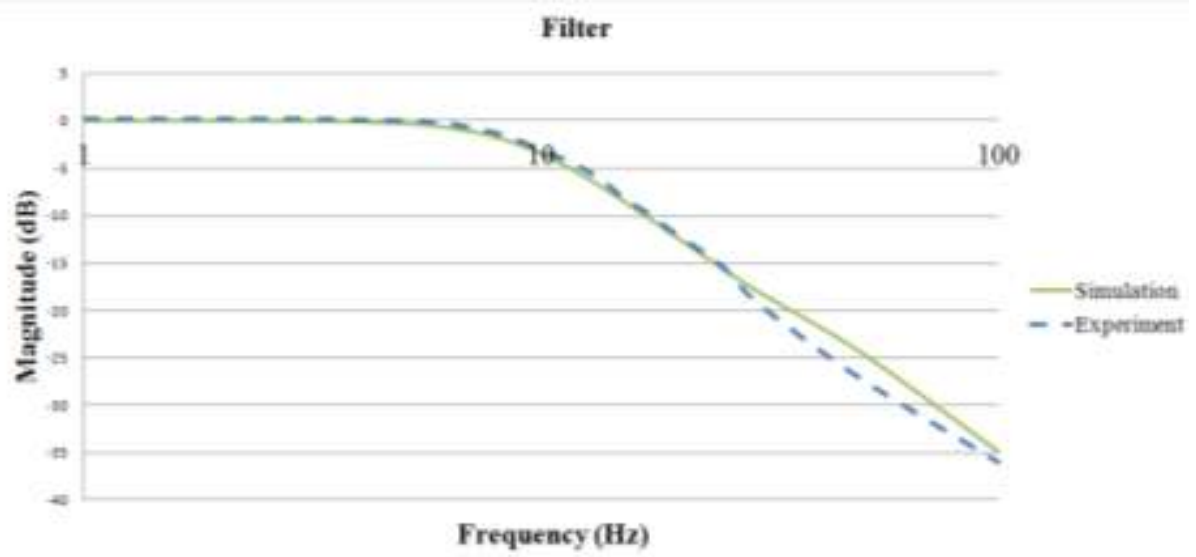

Figure 7 Second-order Sallen Key lowpass filter frequency response.

\subsection{Graphical User Interface (GUI)}

When the user starts the program, a message box is prompted to remind the user to attach the PPG devices before starting the data acquisition. A real-time plot of the two-channel PPG signal is shown in Figure. 7. Figure 8 shows the acquired two-channel PPG signal. Channel 1 is shown in Figure 8 (top) and channel 2 is shown in Figure 8 (bottom). Since the PWTT calculation is beyond the scope of this discussion, its results will not be presented here.

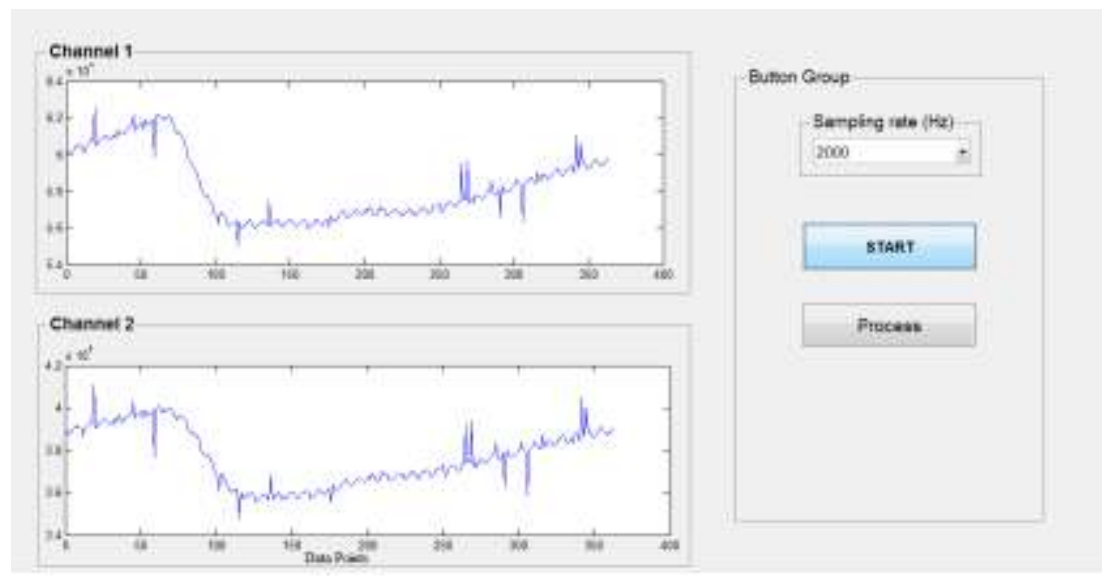

Figure 8 Real-time plot of the two-channel PPG system 
Dual Channel PPG Signal
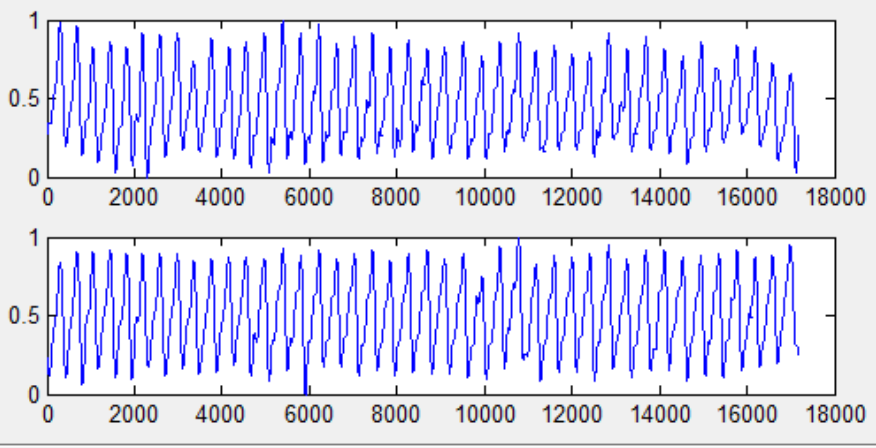

Data Points

Figure 9 Channel 1 (top) and channel 2 (bottom) of the PPG signal

\subsection{Two-Channel Simultaneous PPG Recording Test}

To test the two-channel simultaneous PPG recording system, a sine wave with a $1 \mathrm{~Hz}$ frequency and a $1 \mathrm{Vpk}$-pk was generated using a function generator. It was connected to two ADC inputs and sampled at $2000 \mathrm{kHz}$ (Figure 9). In this experiment, the test frequency was $1 \mathrm{~Hz}$ because this is close to the human heart rate frequency. Note that the noise arising from the ADC switching effect was not visualized after pre-processing. However, the peaks arising from the ADC switching are undesired and will affect the accuracy of the results if the problem remains unsolved. The delay estimation using the cross-correlation technique showed that there was no inter-channel delay between the test signals. The result showed that both channels were simultaneously recorded with the ADC.

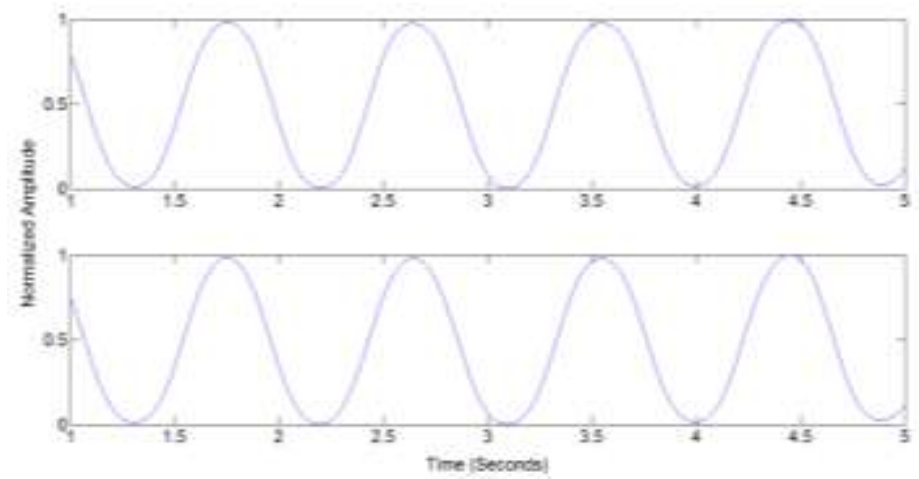

Figure 10 Sine wave $(1 \mathrm{~Hz})$ acquired using the two-channel PPG system at a $2000 \mathrm{~Hz}$ sampling rate. 
During the data acquisition session, the subjects were asked to abstain from consuming food, alcohol and caffeine for at least 4-6 hours prior to the experiment to avoid the vasodilation effect from these substances. Upon arrival, the subjects were asked to rest for 10 minutes to ensure a stable pulse during the data recording session. Figure 10 shows the two-channel PPG signal acquired from the left and the right index finger of a healthy subject using the developed PPG system. The preliminary results show that the delay between the left and the right arm ranged from 4 to $12 \mathrm{~ms}$ in three healthy random subjects. However, the analysis of the PWTT for cardiovascular risk assessment needs more clinical data and medical records for validation.

Besides that, power management is an important issue because the maximum power that can be sourced from the USB is $500 \mathrm{~mW}$. This can only be achieved if there is no sharing of the same USB port. Another observation was that the acquired PPG signals were noisy. This was due to the switching effect of the ADC. The ADS8361 is a successive approximation (SAR) ADC architecture. The switching effect can be eliminated by either replacing it with a delta sigma ADC architecture or adding an analog front-end circuit to the system.

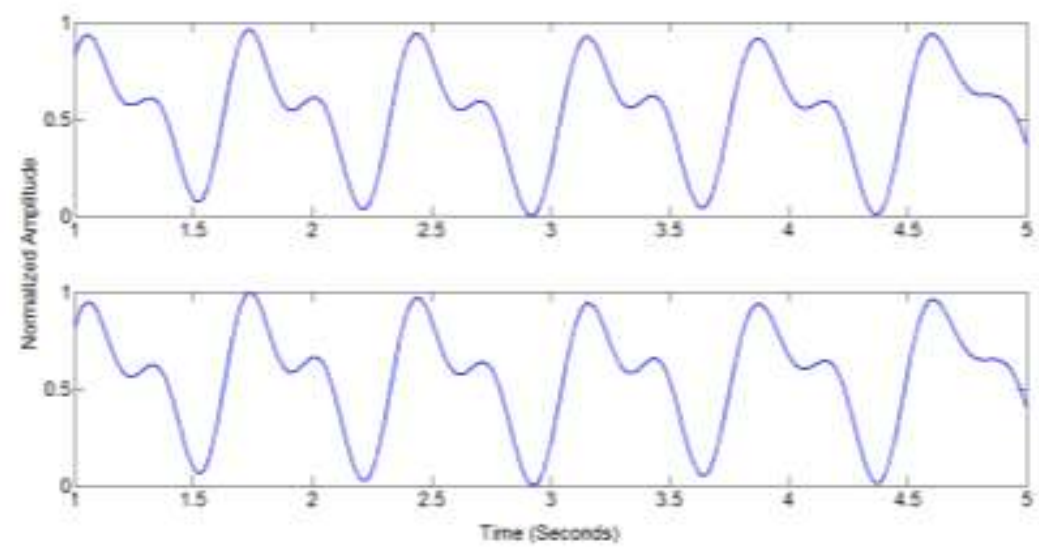

Figure 11 Simultaneous two-channel PPG signal recording from left and right index fingers.

\section{Conclusions}

In this project, a two-channel simultaneous PPG recording system was successfully designed and developed. It is portable, powered by USB port and allows users to do data acquisition in a clinical environment. The crosscorrelation technique was used to estimate the delay between both channels. The sine wave experiment showed that there is no inter-channel delay in the system. Our preliminary results showed that the delay between the left and the 
right arm was from 4 to $12 \mathrm{~ms}$ in three healthy random subjects. However, medical data are required to validate these results and more data need to be acquired to establish the method for cardiovascular risk assessment.

One of the problems observed during the development of the system was the noise problem resulting from the ADC switching effect. As for future work, the system can be improved by using a proper ADC or an analog front-end circuit to avoid this switching effect. The GUI can be improved by adding extra functions such as the determination of the CVD risk. Furthermore, a graphical LCD display can be added to show the CVD risk to the users. This device can be further developed into a cardiovascular risk assessment system for community health screening.

\section{Acknowledgments}

The authors would like to thank Universiti Kebangsaan Malaysia for sponsoring this work under Research University Grant UKM-GUP-2011-352 \& GGPM2011-074.

\section{References}

[1] Zahedi, E., Chellappan, K., Mohd Alauddin, M.A. \& Singh, H, Analysis of the Effect of Ageing on Rising Edge Characteristics of the Photoplethysmogram using a Modified Windkessel Model, Cardiovascular Engineering, 7, pp. 172-181, 2009.

[2] Allen, J., Overbeck, K., Stansby, G. \& Murray, A, Photoplethysmography Assessments in Cardiovascular Disease, Measurement Control, 39, pp. 80-83, 2006.

[3] Allen, J., Oates, C.P., Lees, T.A. \& Murray, A., Photoplethysmography Detection of Lower Limb Peripheral Arterial Occlusive Disease: A Comparison of Pulse Timing, Amplitude and Shape Characteristics, Physiological Measurement, 26, pp. 811-821, 2005.

[4] Allen, J. \& Murray, A., Age-Related Changes in Peripheral Pulse Shape Characteristics at Various Body Sites, Physiological Measurement, 24, pp. 297-307, 2003.

[5] World Health Organization, Cause of death in Malaysia, Geneva: WHO, 2009.

[6] Laurent, S., Boutouyrie, P., Asmar, R., Gautier, I., Laloux, B., Guize, L. Ducimetiere, P. \& Benetos, A., Aortic Stiffness is An Independent Predictor of All-Cause and Cardiovascular Mortality in Hypertensive Patients, Hypertension, 37, pp.1236-1241, 2011.

[7] Oliver, J.J. \& Webb, D.J., Noninvasive Assessment of Arterial Stiffness and Risk of Atherosclerotic Events, Arterioscler Thromb Vasc. Biol., 23, pp. 554-566, 2003. 
[8] Bronzino, J.D, The Biomedical Engineering Handbook, 1, Florida: CRC Press LLC, 2000.

[9] Bhattacharya, P.P., Kanjilal \& Muralidhar, V., Analysis and Characterization of Photoplethysmographic Signal, IEEE Transsaction of Biomedical Engineering, 48, pp. 5-11, 2001.

[10] Allen, J. \& Murray, A., Variability of Photoplethysmography Peripheral Pulse Measurements At The Ears, Thumbs and Toes, IEEE Proceeding Science and Technology, 147(6), pp. 403-407, 2000.

[11] Allen, J., Photoplethysmography and Its Application in Clinical Physiological Measurement, Physiological Measurement, 28, R1, 2007.

[12] Allen, J. \& Murray, A., Similarity in Bilateral Photoplethysmographic Peripheral Pulse Wave Characteristics At The Ears Thumbs and Toes, Physiological Measurement, 21, pp. 369-377, 2000.

[13] Spigulis, J., Erts, R., \& Ozols, M., Optical Multi-Channel Monitoring of Skin Blood Pulsations for Cardiovascular Assessment, SPIE Proc Advanced Biomedical and Clinical Diagnostic Systems, 5318, p.133-139, 2004.

[14] Erts, R., Spigulis, J., Kukulis, I., \& Ozols, M., Bilateral Photoplethysmography Studies of The Leg Arterial Stenosis, Physiological Measurement, 26, pp. 865-874, 2005.

[15] Zahedi, E. \& Mohd. Ali, M.A., Dual-Channel Photoplethysmography Synchronization Using A Barker Sequence, Conference Procedings IEEE Engineering in Medicine and Biology Society, 2, pp. 1952-1955, 2005.

[16] Spigulis, J., Venckus, G. \& Ozols, M., Optical Sensing for Early Cardiovascular Diagnostic, Proceedings SPIE, 3911, pp. 27-31, 2000. 